\title{
Dosimetria Interna em Medicina Nuclear com o Aplicativo GATE: Avaliação do impacto do elemento de dose e do tipo de fonte
}

\author{
Internal Dosimetry in Nuclear Medicine with the application GATE: \\ Evaluation of the impact of dose element and source type
}

\author{
Caroline M. Dartora ${ }^{1}$, Nícollas G. Cavedini ${ }^{1}$, Luis Vinícius de Mouraํㅜ, Ana M. Marques da Silva ${ }^{1}$ \\ ${ }^{1}$ PUCRS, Escola de Ciências, Núcleo de Pesquisa em Imagens Médicas, Porto Alegre, Brasil
}

\begin{abstract}
Resumo
O aplicativo GATE (Geant4 Application for Emission Tomography) disponibiliza uma série de ferramentas que permitem a coleta de dados da interação da radiação com a matéria durante a simulação, como a energia depositada e as partículas criadas dentro de um volume, entre outros. O objetivo deste trabalho é avaliar o impacto do tamanho do elemento de dose na simulação da dose absorvida em um meio atenuador no aplicativo GATE, utilizando fontes pontuais de ${ }^{99 \mathrm{mT}} \mathrm{Tc}$ e ${ }^{18} \mathrm{~F}$. Foi realizada uma análise da influência do tamanho dos elementos do mapa de dose (dosel) na dose absorvida, assim como o impacto de diferentes configurações de fontes. Os resultados mostram que uma matriz com voxels maiores subamostram os valores de dose absorvida, principalmente quando mais próximos da fonte. Assim, para uma dosimetria mais acurada recomenda-se utilizar dosels de menor tamanho próximos da fonte. Em relação à simulação do ${ }^{18} \mathrm{~F}$, as simulações devem ser realizadas com a fonte configurada como "Fluor18" para que todos os processos físicos sejam considerados adequadamente. Conclui-se que o aplicativo GATE é um ambiente confiável para a estimativa de dose em imagens de medicina nuclear, permitindo a investigação e a seleção dos processos de interação da radiação com a matéria mais relevantes para a dosimetria interna.
\end{abstract}

Palavras-chave: dosimetria interna; medicina nuclear; GATE; Mapa de dose; Simulação de Monte Carlo.

\begin{abstract}
For a realistic ${ }^{18} \mathrm{~F}$ simulation of dosimetry purposes, the preconfigured "Fluor18" source, which simulates the positrons emission spectrum, should be used with all physical process of radiation interaction with matter. In conclusion, GATE application is a reliable and friendly environment for dose estimation in nuclear medicine imaging.

GATE application (Geant4 Application for Emission Tomography) provides a series of tools that allow the collection of data from the interaction of radiation with matter during simulation, such as energy deposited and particles created within a volume, among others. The objective of this work is to evaluate the impact of the dose element size on the simulation of the absorbed dose in an attenuating medium in GATE application, using ${ }^{99 m} \mathrm{TC}$ and ${ }^{18} \mathrm{~F}$ point sources. The influence of the dose map elements (dosel) size was investigated on the absorbed dose, as well as the impact of different source configurations. The results show that a matrix with larger voxels underestimates the absorbed dose values, especially when closer to the source. Thus, for a more accurate dosimetry it is recommended to use smaller dosels near the source. In relation to ${ }^{18} \mathrm{~F}$ simulation, they must be performed with the source preset as "Fluor18", so all physical processes can be properly considered. It is concluded that the GATE application is a reliable environment for dose estimation in nuclear medicine imaging, allowing the investigation and selection of the most relevant radiation interaction processes with the material to perform internal dosimetry.
\end{abstract}

Keywords: Internal dosimetry; Nuclear Medicine; GATE; Dose Map, Monte Carlo simulation.

\section{Introdução}

GATE (Geant4 Application for Emission Tomography) é um pacote de ferramentas para a aplicação do Geant4 de simulação de Monte Carlo (MC) para tomografia por emissão. É uma plataforma de código aberto de simulação em MC, que incorpora as bibliotecas do Geant4, em um pacote de ferramentas modular, versátil programável através de scripts, para simulação de imagens médicas, de radioterapia e de dosimetria em um mesmo ambiente ${ }^{1-3}$.
A plataforma GATE permite uma acurada descrição de fenômenos dependentes do tempo, tais como o movimento de detectores, a modelagem da respiração e movimentos cardíacos, assim como a cinética de radiotraçadores ${ }^{1,2}$. Ele permite uma modelagem mais realista dos sistemas de imageamento médico, permitindo a simulação de dados brutos de interação da radiação com o corpo do paciente, permitindo inclusive avaliar a acurácia de algoritmos de reconstrução tomográfica e métodos de correção². Além disso, GATE disponibiliza uma 
série de ferramentas, chamadas atuadores, que permitem a coleta de dados relevantes durante a simulação, como a energia depositada e as partículas criadas dentro de um volume, entre outros. Para a geração do mapa de dose no GATE utiliza-se comando "dose actor", que armazena, em uma matriz 3D, a dose, em Gy.

No GATE, o menor elemento de volume (voxel) dentro da matriz de dose absorvida é chamado de elemento de dose ou dosel, e define a resolução espacial do mapa de dose.

$\mathrm{Na}$ medicina nuclear a fonte radioativa encontra-se dentro do paciente, resultando em uma maior interação da radiação emitida com os tecidos mais próximos à fonte, devido à maior fluência da radiação. Como o número de interações nas regiões próximas à fonte pode ser superior ao número de interações com os detectores mais distantes, o aplicativo GATE permite otimizar a configuração da irradiação, minimizando o número de histórias simuladas através de alterações no dosel. Como o tamanho do dosel pode ser definido pelo usuário, é necessário avaliar o seu impacto na determinação da dose absorvida para diferentes distâncias.

O objetivo deste trabalho foi realizar uma avaliação do impacto do tamanho de elemento de dose na simulação da dose absorvida em um meio atenuador esférico, utilizando fontes pontuais de ${ }^{99 m} \mathrm{Tc}$ e ${ }^{18} \mathrm{~F}$ simuladas no aplicativo GATE.

\section{Materiais e Métodos}

\subsection{Modelagem}

A Figura 1 mostra a primeira geometria modelada utilizada neste trabalho, que foi constituída de uma fonte pontual $\left({ }^{99 m} \mathrm{Tc}\right.$ e $\left.{ }^{18} \mathrm{~F}\right)$, em amarelo, centrada em uma esfera de $15 \mathrm{~cm}$ de raio, em vermelho, preenchida com água. O mundo, estrutura na qual os processos físicos ocorrem, foi definido como uma caixa cúbica de $100 \mathrm{~cm}$ de aresta (em ciano). A segunda geometria utilizada neste trabalho manteve as condições anteriores, somente aumentando o raio da esfera para $30 \mathrm{~cm}$.

As simulações do ${ }^{99 m}$ Tc foram realizadas com a geração de $2 \times 10^{7}$ histórias (aproximadamente $0,5 \mathrm{mCi}$ ), correspondentes à emissão de fótons gama de energia $140 \mathrm{keV}$, de forma a permitir a comparação dos resultados com o trabalho realizado por Papadimitroulas ${ }^{5}$.

A simulação da fonte de ${ }^{18} \mathrm{~F}$ teve atividade definida

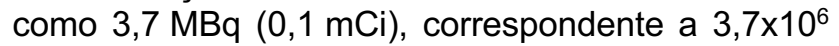
histórias. Neste caso, não foi utilizado o mesmo número de histórias do ${ }^{99 \mathrm{~m}} \mathrm{Tc}$, pois a emissão do ${ }^{18} \mathrm{~F}$ se dá pela emissão de pósitrons, aniquilação e subsequente emissão de dois fótons gama colineares. Foram avaliadas as duas configurações possíveis da fonte de ${ }^{18} \mathrm{~F}$ : "Fluor18" e "back-to-back", em simulações de 1 segundo.

Foram utilizadas as seguintes bibliotecas de interação física: (i) Standard, para efeito fotoelétrico $(E F)$, ionização (I), espalhamento Compton (EC), Bremmstrahlung (B), espalhamentos múltiplos por interações coulombianas (MSC), e aniquilação de pósitrons (A); e (ii) Penelope, para espalhamento Rayleigh (R).

\subsection{Influência do Tamanho do Elemento de Dose}

O aplicativo GATE permite que a matriz na qual é calculada a dose absorvida possa ser definida com tamanhos variáveis de dosel em x, y e z. Neste trabalho, o dosel foi definido como uma série de pequenos cubos (voxels) isotrópicos. Para avaliação da influência do tamanho do elemento de dose, as dimensões do dosel testados foram $0,15 \times 0,15 \times 0,15 \mathrm{~cm}^{3}, \quad 0,20 \times 0,20 \times 0,20 \mathrm{~cm}^{3} \quad \mathrm{e}$ $0,30 \times 0,30 \times 0,30 \mathrm{~cm}^{3}$.

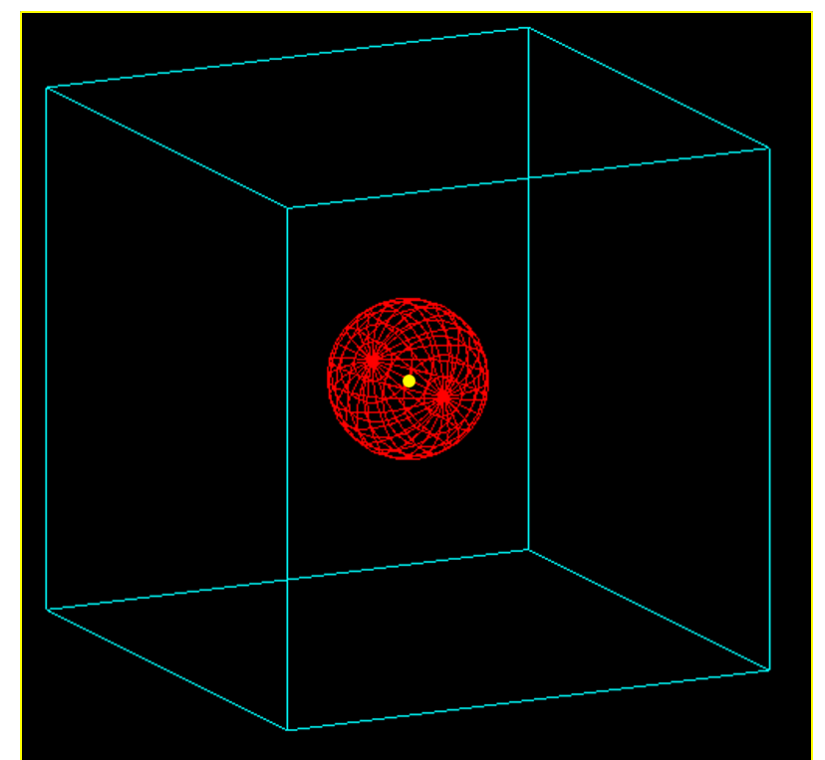

Figura 1. Geometria modelada. Na cor ciano, a caixa do mundo da simulação. Em vermelho, a esfera de $15 \mathrm{~cm}$ de raio com água, na qual é detreminada a dose. No centro da esfera, em amarelo, encontra-se a fonte pontual.

A análise da dose absorvida foi realizada com a técnica de kernel de ponto de dose ou DPK (do inglês, Dose Point Kernel) ${ }^{6}$, que consiste na determinação do valor da energia depositada em cada raio de uma esfera de material conhecido, homogeneamente distribuído, devido a uma fonte pontual centrada emissora de radiação. A distribuição de dose foi calculada em diferentes esferas concêntricas, gerados a partir do centro, conforme a Figura 2.

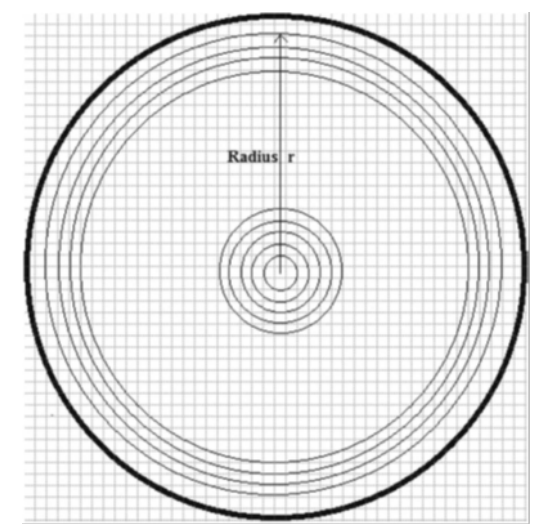

Figura 2. Representação 2D das esferas concêntricos em torno de uma fonte radioativa centrada, nas quais calcula-se a dose absorvida pelo método $\mathrm{DPK}^{5}$. 


\subsection{Configurações da Fonte de ${ }^{18} \mathrm{~F}$}

$O$ aplicativo GATE permite que sejam simuladas duas configurações de fontes para emissão de ${ }^{18} \mathrm{~F}$, configuradas com os nomes: "Fluor18" e "back-toback". Cada tipo de fonte obedece às seguintes características:

- Fluor18: configuração de fonte na qual se define o espectro de energia dos pósitrons emitidos devido ao decaimento do ${ }^{18} \mathrm{~F}$;

- Back-to-back: configuração de fonte na qual são gerados apenas dois fótons de aniquilação com ângulos colineares de $180^{\circ}$.

As duas configurações da fonte de ${ }^{18} \mathrm{~F}$ foram simuladas separadamente para análise da interação dos pósitrons e posterior emissão dos fótons gamas colineares, e, separadamente, somente os fótons gamas colineares. O tamanho de dosel utilizado para construção do mapa de dose para comparação entre os dois modelos de fonte foi de $0,15 \times 0,15 \times 0,15 \mathrm{~cm}^{3}$.

Para uma análise mais detalhada dos processos físicos de interação da radiação com a matéria utilizando a fonte configurada como "Fluor18", foram realizadas simulações de uma fonte pontual de $37 \mathrm{MBq}(0,1 \mathrm{mCi})$, utilizando todas as combinações de configurações de processos físicos descrito na subseção 2.1. Para fins de otimização de tempo computacional, o tamanho de dosel utilizado foi de $0,30 \times 0,30 \times 0,30 \mathrm{~cm}^{3}$.

\section{Resultados}

\subsection{Influência do Tamanho do Elemento de Dose}

\subsubsection{Fonte de ${ }^{99 m} T c$}

A Figura 3 mostra os valores de dose na esfera de água devido a uma fonte pontual centrada de ${ }^{99 \mathrm{~m} T c \text {, }}$ normalizada pelo valor máximo de dose, para cada dose/ simulado, em função da distância da fonte, para os diferentes dosels. As cores representam as diferentes dimensões de dosel simulados: $0,15 \times 0,15 \times 0,15 \mathrm{~cm}^{3}$ $0,20 \times 0,20 \times 0,20 \mathrm{~cm}^{3}$ $0,30 \times 0,30 \times 0,30 \mathrm{~cm}^{3}$ (vermelho).

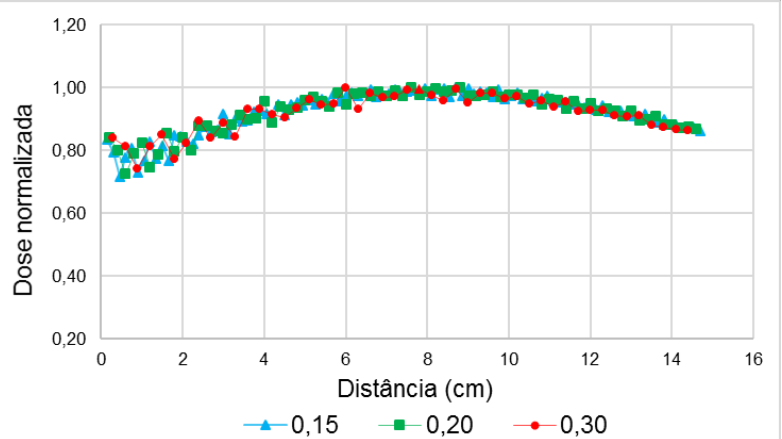

Figura 3. Dose absorvida normalizada para o valor máximo de dose, para a fonte de ${ }^{99 \mathrm{~m} T c} \mathrm{c}$, em função da distância da fonte, para diferentes tamanhos de dosels.

Para fins de comparação com os resultados apresentados por Papadimitroulas ${ }^{5}$ para a simulação de uma fonte de ${ }^{99 m} \mathrm{Tc}$, uma simulação foi realizada com uma esfera de água de $30 \mathrm{~cm}$ de raio, dosel de
$0,30 \mathrm{~cm}$. A Figura 4 mostra o resultado da dose normalizada pelo valor máximo.



Figura 4. Dose absorvida normalizada para o dosel de $0,30 \mathrm{~cm}$ em uma esfera de água de $30 \mathrm{~cm}$ de raio.

\subsubsection{Fonte de ${ }^{18} \mathrm{~F}$}

A Figura 5 mostra os valores de dose absorvida para a fonte de ${ }^{18} \mathrm{~F}$ configurada como "Fluor 18 ", para cada tamanho de dosel avaliado (a) e a dose normalizada para o valor máximo (b). As cores representam os dosels simulados de diferentes dimensões: $0,15 \times 0,15 \times 0,15 \mathrm{~cm}^{3} \quad$ (ciano) $0,20 \times 0,20 \times 0,20 \mathrm{~cm}^{3} \quad$ (verde)

$0,30 \times 0,30 \times 0,30 \mathrm{~cm}^{3}$ (vermelho).

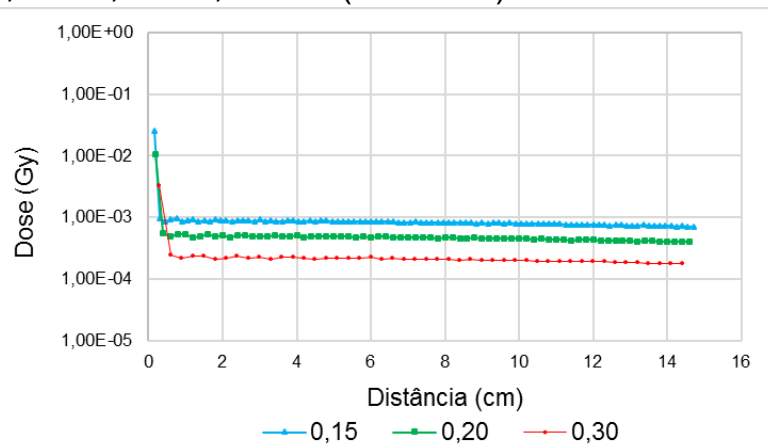

(a)

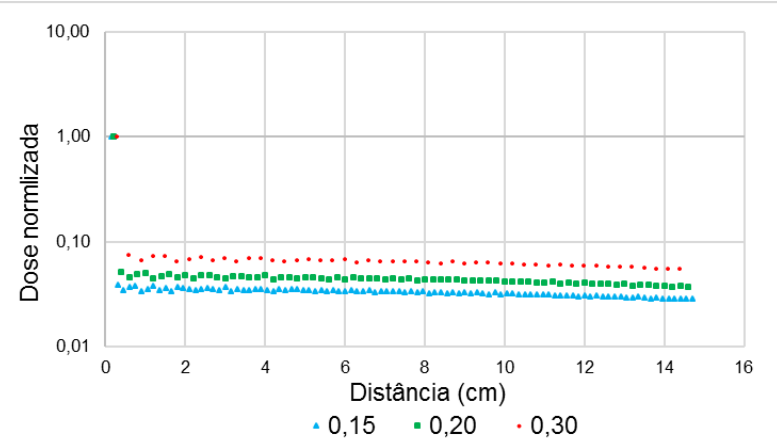

(b)

Figura 5. Doses absorvidas diferentes dosels, para a fonte de ${ }^{18} \mathrm{~F}$, em função da distância da fonte. Em (a) são apresentadas as doses absolutas para cada tamanho de dosel. Em (b), a dose normalizada. $\mathrm{O}$ eixo y está em escala logarítimica.

Para melhor visualização do comportamento da curva de dose absorvida para a fonte configurada como "Fluor18", uma simulação foi realizada com uma esfera de água de $30 \mathrm{~cm}$ de raio, dosel de 0,30 $\mathrm{cm}$. A Figura 6 mostra em (a) a dose normalizada com o primeiro ponto de dose e em (b), sem o primeiro ponto, evidenciando o comportamento da curva ao longo da distância. 


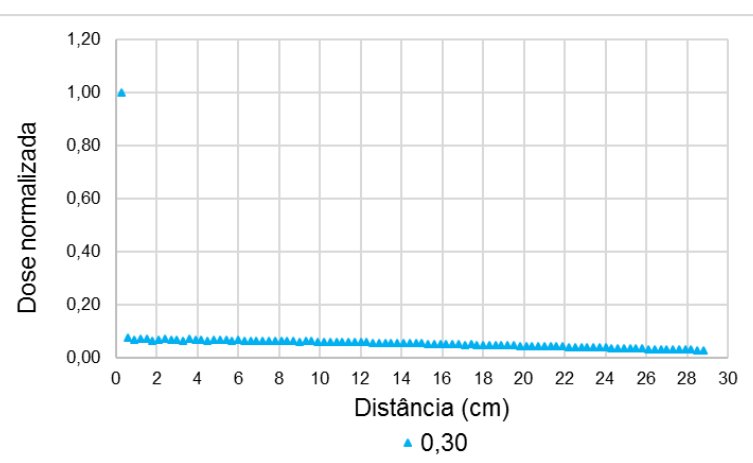

(a)

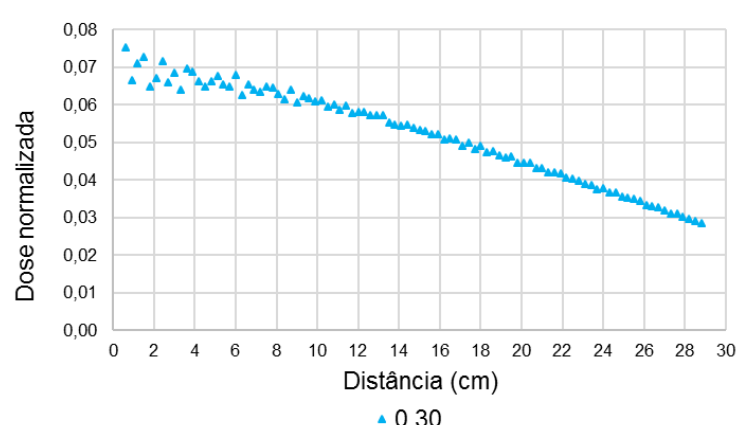

(b)

Figura 6. Doses absorvidas normalizadas para o dose/ de 0,30 $\mathrm{cm}$, para a fonte de ${ }^{18} \mathrm{~F}$, em uma esfera de raio $30 \mathrm{~cm}$. Em (a) a dose normalizada com o primeiro ponto de dose e em (b), sem o primeiro ponto, destacando o comportamento da curva.

\subsection{Interação de fontes emissoras de pósitrons} $\left({ }^{18} \mathrm{~F}\right)$ com a matéria

A Figura 7 apresenta as doses absorvidas, em Gy, em função da distância do centro da esfera, com dosel de $0,15 \mathrm{~cm}$, quando são utilizados os diferentes tipos de configuração da fonte de ${ }^{18} \mathrm{~F}$ no aplicativo GATE: "Fluor18"e "back-to-back". Para evidenciar as diferenças, foi utilizada uma escala logarítmica de dose. A seta vermelha destaca o primeiro ponto de deposição de dose, que surge apenas quando é utilizada a configuração "Fluor 18".

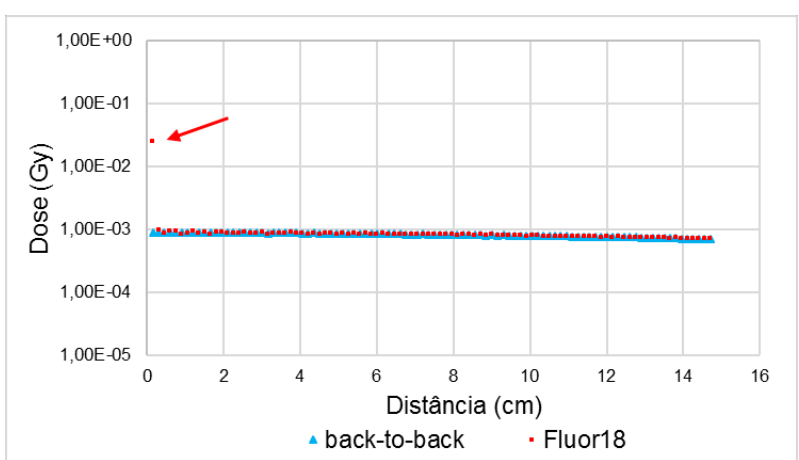

Figura 7. Doses simuladas em uma esfera de água para ${ }^{18} \mathrm{~F}$ definida como "back-to-back" (ciano) e "Fluor18" (laranja). A seta vermelha aponta a dose no primeiro dosel da fonte

"Fluor18". O eixo vertical está em escala logarítmica.

A diferença percentual entre a dose nas simulações com as duas configurações de fonte de ${ }^{18} \mathrm{~F}$ na Figura 7 é de $23 \%$. Desconsiderando a dose referente ao dosel mais próximo a fonte, a diferença percentual entre as dose com as duas configurações de ${ }^{18} \mathrm{~F}$ cai para $0,45 \%$.
Para a fonte configurada "Fluor18", os seguintes processos físicos não apresentaram deposição de energia na esfera: aqueles associados à aniquilação individualmente e associados aos processos de Bremsstrahlung (B), espalhamento Rayleigh (R) e MSC.

Processos físicos que combinavam aniquilação e ionização tiveram deposição de energia somente na primeira esfera central do DPK, onde encontra-se a fonte de ${ }^{18} \mathrm{~F}$.

O processo de ionização do meio apresentou uma contribuição de $23 \%$ da dose total na esfera. No primeiro ponto de dose, essa contribuição é maior, sendo $92,6 \%$ do total de dose encontrado.

A fim de verificar a contribuição efetiva do espalhamento Compton para a deposição de energia, este processo físico foi associado ao processo de aniquilação e ionização. A Erro! Fonte de referência não encontrada. apresenta, na curva em verde, a dose quando ocorre somente o processo de aniquilação de pósitrons associado ao espalhamento Compton. Em preto, encontra-se a distribuição de dose para quando todos os processos físicos de interação da radiação com a matéria ocorrem, para fins de comparação.



Figura 8. Dose absorvida devido a todos os processos (linha preta) para a fonte "Fluor18". Em verde, processo de deposição de energia sem ocorrência de ionização do meio e somente com espalhamento Compton (EC) habilitado.

A dose depositada devido ao espalhamento Compton após a aniquilação representa $40,4 \%$ da contribuição na deposição de energia, principalmente após a metade do raio da esfera $(7,5 \mathrm{~cm})$. Quando os processos de aniquilação e espalhamento Compton são associados à ionização, a contribuição individual do EC aumenta para $98,6 \%$ da dose total.

Quando a contribuição de dose é simulada somente com processo de interação de radiação sem carga com a matéria $(A+E C+E F+R)$, obtém-se que o espalhamento Rayleigh ( $R$ ) contribui com cerca de $0,004 \%$ da dose. O efeito fotoelétrico representa $0,004 \%$. Ao associar o processo de ionização do meio com espalhamento Compton e efeito fotoelétrico, o EF representa $1,4 \%$ da dose depositada.

$O$ efeito fotoelétrico, associado a outros processos físicos de interação da radiação com a matéria, foi simulado com e sem a participação do espalhamento Compton. Sem espalhamento Compton, as doses devido ao efeito fotoelétrico, após só aniquilação, e aniquilação e ionização do pósitron emitido pela fonte 
na dose total foram de $0,002 \%$ e $23 \%$, respectivamente.

\section{Discussão}

\subsection{Influência do Elemento de Dose}

\subsubsection{Fonte de ${ }^{99 m} T c$}

Observa-se um comportamento semelhante para a dose normalizada em função da distância, para todos os dosels utilizados para o ${ }^{99 \mathrm{~m} T c}$, após uma distância aproximadamente igual à metade do raio da esfera (Figura 3 e 4). Assim, o tamanho do dosel não afeta o cálculo de dose de maneira significativa para a fonte emissora de radiação gama. No entanto, os valores de dose normalizada apresentam maior variabilidade nas proximidades da fonte, para todos os tamanhos de dosel, principalmente nos primeiros $4 \mathrm{~cm}$. Este comportamento se dá devido ao pequeno número de elementos que compõem o cálculo de dose as cascas esféricas de pequeno raio. Este efeito de variação devido à amostragem desaparece à medida que as cascas esféricas de maior raio, que possuem maior número de elementos. Tais resultados de dose são compatíveis com aqueles encontrados por outros pesquisadores em simulações da emissão de radionuclídeos com o aplicativo GATE ${ }^{4,5}$.

A matriz com dosels de maior dimensão subamostram os valores de dose, principalmente nas posições mais próximas da fonte. Assim, para uma dosimetria mais acurada, é recomendável utilizar dosels de menor tamanho nos pontos mais próximos da fonte. No caso de necessidade de diminuir o tempo computacional da simulação, sugere-se utilizar tamanhos variáveis de dosel, com um tamanho de dosel maior para pontos mais distantes da fonte.

\subsubsection{Configurações da Fonte de ${ }^{18} \mathrm{~F}$}

Observa-se um comportamento semelhante da dose absorvida em todos os dosels utilizados para a simulação do ${ }^{18} \mathrm{~F}$, configurada para "Fluor18", que considera a emissão de pósitrons (Figura 5). Devido à forte interação dos pósitrons com o meio, existe uma alta deposição de energia antes do primeiro centímetro de distância da fonte.

No entanto, os valores da dose absorvida na esfera de água para a fonte de ${ }^{18} \mathrm{~F}$ são significativamente distintos para diferentes tamanhos de dosels. Dentre as hipóteses para explicar este comportamento está o fato de que a dose em cada dosel é calculada pela média de interações ocorridas dentro do volume. Dessa forma, como o número de interações é aproximadamente o mesmo dentro das dimensões dos dosels avaliados, quanto menor o volume do dosel, maior será o número de interações por unidade de volume, gerando um maior valor de dose total no menor dosel, como mostra a Figura 5(a). Esse efeito é realçado pelo fato desta fonte ser configurada com a emissão de pósitrons, que geram alta dose no primeiro dose. Assim, a dose no primeiro ponto próximo da fonte é maior para o dosel de $0,15 \times 0,15 \times 0,15 \mathrm{~cm}^{3}$ do que para 0 dosel de $0,20 \times 0,20 \times 0,20 \mathrm{~cm}^{3}$, e para o dosel de $0,30 \times 0,300,30 \mathrm{~cm}^{3}$ (Figura 5(a)). Maiores estudos devem ser realizados para avaliar tais hipóteses. Mas, pode-se assumir que para uma melhor amostragem, o menor dosel deve ser utilizado nas simulações próximas à fonte.

Adicionalmente, as diferentes configurações da fonte de ${ }^{18} \mathrm{~F}$ no aplicativo GATE mostram um grande impacto na dose. A fonte configurada como "Fluor18" mostra que uma alta deposição de dose é realizada próximo à fonte (Figura $6 \mathrm{a}$ ), resultante da interação dos pósitrons e um comportamento de deposição de energia mais lento a partir do segunda esfera concêntrica (Figura 6 b), resultante da interação com a radiação gama de aniquilação. Os pósitrons, por serem partículas carregadas, interagem com o meio rapidamente depositando sua energia principalmente por ionização nos pontos mais próximos à fonte, o que se mostra coerente com o alcance médio dos pósitrons $\left(R_{\text {mean }}=0,6 \mathrm{~mm} \text { e } R_{\max }=2,4 \mathrm{~mm}\right)^{7}$. A dose devido à ionização, após aniquilação, é depositada antes dos primeiros $2 \mathrm{~cm}$ de distância da fonte, o que é mostrado no primeiro ponto da curva vermelha (seta vermelha) da Figura 7. Na configuração "back-toback" este efeito de deposição alta de dose próximo à fonte não é observado nos resultados da simulação, pois esta configuração considera apenas a emissão de fótons de aniquilação.

A diferença percentual entre a dose total nas duas configurações de fonte de ${ }^{18} \mathrm{~F}$ é de $23 \%$. Apesar dessa diferença, o uso da fonte configurada "back-toback" para o ${ }^{18} \mathrm{~F}$ pode ser utilizado para simulaçõsde imagens de tomografia por emissão de pósitrons ou para estimar a deposição de energia em tecidos alvo distantes do tecido fonte, sem prejudicar significativamente os resultados, visto que a diferença cai para $0,45 \%$ para distância maiores do que alguns milímetros da fonte. A deposição de dose nos tecidos fontes, no entanto, deve levar em conta essa contribuição da dose devido aos pósitrons. A dose nas proximidades da fonte deve-se basicamente ao processo físico de ionização, enquanto pontos mais distantes, o processo preponderante é o espalhamento Compton, concordando com os achados para a faixa de energia dos fótons colineares emitidos e o número atômico do material utilizado na simulação ${ }^{8}$.

Os estudos iniciais realizados com o aplicativo GATE com fontes e objetos atenuadores simples mostraram que existe a possibilidade de determinar a dose interna com a utilização de dose/s de diferentes tamanhos nas simulações. Quanto à simulação de fontes emissoras de pósitrons no aplicativo GATE sugere-se $\mathrm{o}$ uso da fonte pré-configurada como "Fluor18" para que a simulação inclua as contribuições de dose tanto da interação dos pósitrons, quanto dos fótons gama resultantes da aniquilação, particularmente nos estudos de dosimetria interna.

\section{Conclusões}

Este estudo mostrou que o tamanho do voxel a ser utilizado nas dosimetria pela técnica DPK apresenta as maiores diferenças na dose absorvida quando os pontos avaliados estão mais próximos da fonte, tanto 
para a fonte de ${ }^{99 m} \mathrm{Tc}$ quanto para a fonte de ${ }^{18} \mathrm{~F}$. A influência do tamanho do dosel torna-se desprezível a maiores distâncias. Para uma dosimetria mais acurada sugere-se que o tamanho do dosel seja o menor possível. Para viabilizar as simulações e diminuir o tempo computacional, sugere-se utilizar diferentes tamanhos de dosels para diferentes distâncias da fonte.

Apesar de sugerir a escolha do tipo de fonte para ${ }^{18} \mathrm{~F}$ como "Fluor18", para fins de estudo da interação da radiação com a matéria, a configuração da fonte como back-to-back, que simula somente a emissão de fótons colineares, pode ser de grande interesse para a simulação de imagens e para a dosimetria de órgãos distantes do órgão fonte.

Quanto ao comportamento dos processos físicos de interação da radiação com a matéria na deposição de energia na água por uma fonte de ${ }^{18} \mathrm{~F}$, percebe-se que após forte interação inicial do pósitron com o meio por ionização, o espalhamento Compton tornase predominante nos processos de interação da radiação com a matéria.

Dentre as limitações deste estudo destaca-se os erros gerados pelas incertezas estatísticas intrínsecas à abordagem de simulação de Monte Carlo, que é fortemente dependente do número de eventos simulados. Para trabalhos futuros, pretendese ampliar o número de histórias simuladas, exigindo um esforço de paralelizaçao das simulações computacionais. Adicionalmente, pretende-se realizar uma análise da influência do tamanho do dosel em regiões mais próximas à fonte na determinação da dose absorvida.

\section{Referências}

1. ASSIÉ, K. et al. Monte Carlo simulation in PET and SPECT instrumentation using GATE. Nuclear Instruments and Methods in Physics Research Section A: Accelerators, Spectrometers, Detectors and Associated Equipment, v. 527, n. 1-2, p. 180-189, jul. 2004.

2. LOUDOS, George K. Monte Carlo simulations in Nuclear Medicine. In: GRANJA, Carlos; LEROY, Claude; STEKL, Ivan (Ed.). AIP Conference Proceedings. AIP, 2007. p. 147-150.

3. SARRUT, D. et al. A review of the use and potential of the GATE Monte Carlo simulation code for radiation therapy and dosimetry applications. Medical Physics, v. 41, n. 6, p. 064301, jun. 2014.

4. LOUDOS, G. et al. A radionuclide dosimetry toolkit based on material specific Monte Carlo dose kernels. Nuclear Medicine Communications, v. 30, 2009.

5. PAPADIMITROULAS, Panagiotis et al. Photon dose kernels dataset for nuclear medicine dosimetry, using the GATE Monte Carlo toolkit. In: Nuclear Science Symposium and Medical Imaging Conference (NSS/MIC), 2011 IEEE. IEEE, 2011. p. 1390-1394.

6. PAPADIMITROULAS, P.; LOUDOS, G. K.; NIKIFORIDIS, G.; KAGADIS, G.C. A dose point kernel database using GATE Monte Carlo simulation toolkit for nuclear medicine applications: comparison with other Monte Carlo codes. Medical Physics, v. 39, n. 8, p. 5238-5247, Aug. 2012.

7. ALVA-SANCHEZ, $H$. et al. Positron range in tissue-equivalent materials: experimental microPET studies. Physics in Medicine \& Biology, v. 61, n. 17, p. 6307, 2016.

8. ATTIX, Frank Herbert. Introduction to radiological physics and radiation dosimetry. John Wiley \& Sons, 2008.
E-mail: caroline.dartora@acad.pucrs.br

\section{Contato:}

Caroline Machado Dartora

Av. Ipiranga, 6681 - Partenon, Porto Alegre, RS. 